\section{E-009 COMPLICATIONS IN STENTING WITH OR WITHOUT COILING FOR TREATMENT OF ACUTELY RUPTURED CEREBRAL ANEURYSMS}

G Wong*. Neurosurgery, The Chinese University of Hong Kong, Hong Kong

\subsection{6/neurintsurg-2019-SNIS.84}

Objective The purpose of this study was to report the authors' experiences in stenting for ruptured cerebral aneurysms in the acute period and to evaluate the hemorrhagic and ischemic complications and risk factors.

Methods Between January 2013 and October 2018, fiftyfour patients had stenting with or without coiling for treatment of acutely ruptured cerebral aneurysms that were not amendable to coiling alone. Hospital notes were retrospectively reviewed. All patients had a dose of intravenous abciximab $10 \mathrm{mg}$ and heparin 2000 unit before stenting and dual antiplatelet loading and daily treatment started after procedure.

Results Of the 54 patients, twenty-nine (54\%) had braided stents, nine (17\%) had laser-cut stents, and 16(30\%) had flow diverters. Thirty-seven (69\%) had also coiling and 17(31\%) had stenting alone. There was $2(4 \%)$ rebleed in two patients with braided stent and partial embolization resulting in mortality. There were three $(6 \%)$ procedure-related cerebral infarction. There were $3(6 \%)$ significant intraventricular hemorrhage and intracerebral hemorrhage.

Conclusions In patients undergoing stenting for acutely ruptured cerebral aneurysms, partial embolization and ICP catheter insertion were risk factors for complications and mortality. Procedure-related infarction rate was 6\%. Stenting could be considered as an alternative treatment strategy for acutely ruptured cerebral aneurysms not amendable to coiling alone.

Disclosures G. Wong: 1; C; Medtronic.

\section{E-010 RELATION BETWEEN BRAIN NATREURETIC PEPTIDE AND DELAYED CEREBRAL ISCHEMIA IN PATIENTS WITH ANEURYSMAL SUBARACHNOID HEMORRHAGE}

${ }^{1} \mathrm{G}$ Kaur*, ${ }^{2} \mathrm{~N}$ Damodara, ${ }^{2} \mathrm{R}$ Gupta, ${ }^{1} \mathrm{~V}$ Patel, ${ }^{1} \mathrm{~J}$ Santarelli, ${ }^{1} \mathrm{C}$ Gandhi, ${ }^{1} \mathrm{~F}$ Al-Mufti. ${ }^{1}$ Neurosurgery, Westchester Medical Center, Valhalla, NY; ${ }^{2}$ Rutgers University, Newark, NJ

\subsection{6/neurintsurg-2019-SNIS.85}

Background Immune regulation and inflammation is implicated in the development of delayed cerebral ischemia (DCI) in patients with aneurysmal subarachnoid hemorrhage (aSAH). BNP is implicated in fluid dysregulation and inflammation in critically-ill patients. We explored the association between BNP levels and development of DCI in patients with a SAH.

Methodology Patients were enrolled from the Neurological Intensive Care Unit between 2006 and 2015 in the SAH Outcomes Project, a single center, prospective, observational cohort study. Demographic data, treatment and outcomes, and BNP levels at admission and through the hospital admission were noted.

Results In the 149 patients included in the analysis, 79 developed DCI during their hospital course. Significantly higher number of patients in the DCI group had external ventricular drain $(E V D)$ placements $(p=0.008)$. The DCI group also had significantly higher rates of rebleeding and hydrocephalus. In the logistic regression analysis between BNP levels and DCI, there is a significant association with admission BNP and DCI (OR-1.002, 95\%CI 1.00-1.004, $\mathrm{p}=0.017$ ), DCI and highest BNP (OR 1.001, 95\%CI-1.0011.002, $\mathrm{p}=0.002)$, and change in BNP with change in time (OR 1.006, 95\%CI 1.002-1.01, $\mathrm{p}=0.002$ ). The ROC curve analysis for DCI based on BNP showed that the highest BNP level during hospital admission (AUC 0.78) was a stronger predictor than the change in BNP over time (AUC 0.776) or the admission BNP (AUC 0.632).

Conclusion There is a significant association between the BNP level and the risk of developing DCI. This increased risk for DCI is associated not only with higher baseline BNP values (admission BNP) but also with the highest BNP level attained during the hospital course as well as the rapidity of change or increase in BNP over time. Hence, BNP values may help to identify SAH patients at high risk of cardiac morbidity and facilitate appropriate triaging.

Disclosures G. Kaur: None. N. Damodara: None. R. Gupta: None. V. Patel: None. J. Santarelli: None. C. Gandhi: None. F. Al-Mufti: None.

\section{E-011 PREDICTORS OF CLINICAL OUTCOME AFTER TREATMENT OF INTRACRANIAL ANEURYSMS WITH THE PIPELINE EMBOLIZATION DEVICE (PED)}

A Griffin*, E Hauck. Duke University Medical Center, Durham, NC

\subsection{6/neurintsurg-2019-SNIS.86}

Introduction Flow-diverting stents have revolutionized the endovascular treatment of intracranial aneurysms. The purpose of this study is to identify predictors of adverse outcomes associated with the pipeline embolization device (PED).

Materials and methods A retrospective analysis was performed of all patients treated with PED at a single high-volume center from January 2014 to September of 2018. Clinical outcomes, neurological morbidity/mortality and additional variables were analyzed.

Results 204 aneurysms in 170 patients were treated with PED. Mean length of follow up was 11 months. 181 aneurysms $(89 \%)$ were located in the anterior circulation, 23 (11\%) were found in the posterior circulation. The majority of the aneurysms were saccular (82\%), followed by fusiform (11\%), blister (4\%) and dissecting pseudoaneurysms (3\%). Mean aneurysm size was $8.2+5.7 \mathrm{~mm}$ with $145(71 \%)$ small aneurysms (< $10 \mathrm{~mm}), 53(26 \%)$ large aneurysms (10-24 mm), and $6(3 \%)$ giant aneurysms $(\geq 25 \mathrm{~mm})$. 92\% of aneurysms were unruptured, $8 \%$ were ruptured. Neurological symptoms lasting less than 7 days were considered minor. The overall major neurological morbidity/mortality was $4.7 \%$ and $1.8 \%$ respectively. The all-cause mortality was $2.9 \%$. After Bonferroni correction for multiple testing (significance at 0.004 level), the only significant predictors of neurological morbidity/mortality included the baseline modified Rankin Scale (mRS; $\mathrm{p}=0.001)$ and aneurysm neck size $(p=0.003)$. Aneurysm size $(p=0.006)$, rupture at presentation (0.006) anterior versus posterior location $(p=0.02)$ did not reach significance for multiple testing. The Platelet Reactivity Unit (PRU), parent vessel diameter and patient age were not associated with adverse events.

Conclusions The PED has a satisfactory safety profile in experienced hands, both on- and off-label. Nevertheless, a poor 